\title{
Rogue Waves of the Kundu-Nonlinear Schrödinger Equation
}

\author{
Chengchuang Zhang, Chuanzhong Li, Jingsong He \\ Department of Mathematics, Ningbo University, Ningbo 315211, China \\ Email: 360112471@qq.com
}

Received 2013

\begin{abstract}
This paper is based on the Darboux transformation of the Kundu-Nonlinear Schrödinger equation. The rogue wave solutions are obtained from periodic seed solutions. After that, the higher order rogue wave solutions of the Kundu-Nonlinear Schrödinger equation are given. Finally, we show that free parameters in eigenfunctions can adjust the patterns of the higher order rogue waves.
\end{abstract}

Keywords: Darboux Transformation; Rogue Waves; Kundu-Nonlinear Schrödinger Equation

\section{Introduction}

It is remarked that the Darboux transformation is an efficient method to generate the soliton solutions for integrable equations [1]. The determinant representation of n-fold Darboux transformation of AKNS system was given in [2]. Recently, the rogue waves observed firstly in the ocean [3], have been also studied extensively in several fields, such as optical system [4], water tanks[5], Bose-Einstein condensate [6-7],space plasma [8], and even in a financial system [9]. It can be well-described by the analytical expressions for the spectra of breather solutions at the zero point. Furthermore there are several interesting patterns of the higher order rogue waves [10]. In this paper, we shall study the rogue waves of the Kundu-Nonlinear Schrödinger(Kundu-NLS) equation,

$$
i Q_{t}+Q_{x x}-2 \alpha Q^{2} Q^{*}-\left(\theta_{t}+\theta_{x}^{2}-i \theta_{x x}\right) Q+2 i \theta_{x} Q_{x}=0,(1)
$$

where $\theta$ is a arbitrary gauge function, $Q^{*}$ denotes the complex conjugate of $Q$.

In Section 2, the Lax representation and two-fold Darboux transformation of the Kundu-NLS equation will be given. In Section 3, From a periodic seed solution, breather solutions, the first-order rogue wave and higher order rogue wave solutions of the Kundu-NLS equation are given. The higher order rogue waves can be separated into some different types by adjusting free parameters in eigenfunctions. The final Section is a short summary.

\section{Lax Representation and 2-fold Darboux Transformation of the Kundu-NLS Equation}

Now, we concentrate on the Kundu-NLS equation.
The linear eigenvalue problem of Kundu-NLS equation can be expressed in the form of Lax pair $M$ and $N$ as

$$
\begin{aligned}
& \Phi_{x}=M \Phi=\left(-i \lambda \sigma_{3}+\sqrt{\alpha} V_{0}\right) \Phi, \\
& \Phi_{t}=N \Phi=\left(-2 i \lambda^{2} \sigma_{3}+2 \sqrt{\alpha} V_{0}+V_{1}\right) \Phi,
\end{aligned}
$$

with

$$
\begin{gathered}
\sigma_{3}=\left(\begin{array}{cc}
1 & 0 \\
0 & -1
\end{array}\right), V_{0}=\left(\begin{array}{cc}
0 & Q e^{i \theta} \\
-Q^{*} e^{-i \theta} & 0
\end{array}\right), \\
V_{1}=\left(\begin{array}{cc}
\alpha i|Q|^{2} & \sqrt{\alpha i}\left(Q e^{i \theta}\right)_{x} \\
\sqrt{\alpha} i\left(Q^{*} e^{-i \theta}\right)_{x} & -\alpha i|Q|^{2}
\end{array}\right) .
\end{gathered}
$$

Here $\lambda$, an arbitrary complex number, is called the eigenvalue(or spectral parameter), $Q^{*}$ denotes the complex conjugate of $Q, \theta$ is a arbitrary gauge function, $\alpha$ is a real parameter and $\Phi$ is called the eigenfunction associated with $\lambda$ of the Kundu-NLS equation. Firstly, let us consider a matrix $T$ of gauge transformation for the spectral problem (2) and (3) with the following form $\Phi^{[1]}=T \Phi$. New function $\Phi^{[1]}$ is supposed to satisfy $\Phi_{x}^{[1]}=M^{[1]} \Phi^{[1]}, \Phi_{t}^{[1]}=N^{[1]} \Phi^{[1]}$, then matrix $T$ should satisfy following identities $T_{x}+T M=M^{[1]} T$, $T_{t}+T N=N^{[1]} T$, we obtain

$$
M_{t}^{[1]}-N_{x}^{[1]}+\left[M^{[1]}, N^{[1]}\right]=T\left(M_{t}-N_{x}+[M, N]\right) T^{-1} .
$$

This implies that, under the transformation $\Phi^{[1]}=T \Phi$, it is crucial to construct a matrix $T$ so that $M^{[1]}$ and $N^{[1]}$ have the same forms as that $M$ and $N$. At the same time, the old potentials(or seed solutions) in spectral matrixes $M$ and $N$ are mapped into new potentials(or new solutions) in terms of transformed spectral matrixes 
$M^{[1]}$ and $N^{[1]}$. With the help of symbolic computation, MAPLE, after some analysis and some calculations, the two-fold Darboux transformation of Kundu-NLS equation can be represented as follows:

$$
\begin{aligned}
T_{2}\left(\lambda ; \lambda_{1}, \lambda_{2}, \lambda_{3}, \lambda_{4}\right) & =\lambda^{2} I+t_{1}^{[2]} \lambda+t_{0}^{[2]} \\
= & \frac{1}{\Delta_{2}}\left(\begin{array}{ll}
\left(\Pi_{2}\right)_{11} & \left(\Pi_{2}\right)_{12} \\
\left(\Pi_{2}\right)_{21} & \left(\Pi_{2}\right)_{22}
\end{array}\right)
\end{aligned}
$$

where

$$
\begin{aligned}
\Delta_{2}=\left|\begin{array}{llll}
\Phi_{1,1} & \Phi_{1,2} & \lambda_{1} \Phi_{1,1} & \lambda_{1} \Phi_{1,2} \\
\Phi_{2,1} & \Phi_{2,2} & \lambda_{2} \Phi_{2,1} & \lambda_{2} \Phi_{2,2} \\
\Phi_{3,1} & \Phi_{3,2} & \lambda_{3} \Phi_{3,1} & \lambda_{3} \Phi_{3,2} \\
\Phi_{4,1} & \Phi_{4,2} & \lambda_{4} \Phi_{4,1} & \lambda_{4} \Phi_{4,2}
\end{array}\right|, \\
\left(\Pi_{2}\right)_{11}=\left|\begin{array}{ccccc}
1 & 0 & \lambda & 0 & \lambda^{2} \\
\Phi_{1,1} & \Phi_{1,2} & \lambda_{1} \Phi_{1,1} & \lambda_{1} \Phi_{1,2} & \lambda_{1}^{2} \Phi_{1,1} \\
\Phi_{2,1} & \Phi_{2,2} & \lambda_{2} \Phi_{2,1} & \lambda_{2} \Phi_{2,2} & \lambda_{2}^{2} \Phi_{2,1} \\
\Phi_{3,1} & \Phi_{3,2} & \lambda_{3} \Phi_{3,1} & \lambda_{3} \Phi_{3,2} & \lambda_{3}^{2} \Phi_{3,1} \\
\Phi_{4,1} & \Phi_{4,2} & \lambda_{4} \Phi_{4,1} & \lambda_{4} \Phi_{4,2} & \lambda_{4}^{2} \Phi_{4,1}
\end{array}\right|, \\
\left(\Pi_{2}\right)_{12}=\left|\begin{array}{ccccc}
0 & 1 & 0 & \lambda & 0 \\
\Phi_{1,1} & \Phi_{1,2} & \lambda_{1} \Phi_{1,1} & \lambda_{1} \Phi_{1,2} & \lambda_{1}^{2} \Phi_{1,1} \\
\Phi_{2,1} & \Phi_{2,2} & \lambda_{2} \Phi_{2,1} & \lambda_{2} \Phi_{2,2} & \lambda_{2}^{2} \Phi_{2,1} \\
\Phi_{3,1} & \Phi_{3,2} & \lambda_{3} \Phi_{3,1} & \lambda_{3} \Phi_{3,2} & \lambda_{3}^{2} \Phi_{3,1} \\
\Phi_{4,1} & \Phi_{4,2} & \lambda_{4} \Phi_{4,1} & \lambda_{4} \Phi_{4,2} & \lambda_{4}^{2} \Phi_{4,1}
\end{array}\right|, \\
\begin{array}{lllll}
1 & 0 & \lambda & 0 & 0 \\
\Phi_{1,1} & \Phi_{1,2} & \lambda_{1} \Phi_{1,1} & \lambda_{1} \Phi_{1,2} & \lambda_{1}^{2} \Phi_{1,1} \\
\Phi_{2,1} & \Phi_{2,2} & \lambda_{2} \Phi_{2,1} & \lambda_{2} \Phi_{2,2} & \lambda_{2}^{2} \Phi_{2,2} \\
\Phi_{3,1} & \Phi_{3,2} & \lambda_{3} \Phi_{3,1} & \lambda_{3} \Phi_{3,2} & \lambda_{3}^{2} \Phi_{3,2} \\
\Phi_{4,1} & \Phi_{4,2} & \lambda_{4} \Phi_{4,1} & \lambda_{4} \Phi_{4,2} & \lambda_{4}^{2} \Phi_{4,2}
\end{array} \mid, \\
\begin{array}{lllll}
0 & 1 & 0 & \lambda & \lambda^{2} \\
\Phi_{1,1} & \Phi_{1,2} & \lambda_{1} \Phi_{1,1} & \lambda_{1} \Phi_{1,2} & \lambda_{1}^{2} \Phi_{1,1} \\
\Phi_{2,1} & \Phi_{2,2} & \lambda_{2} \Phi_{2,1} & \lambda_{2} \Phi_{2,2} & \lambda_{2}^{2} \Phi_{2,2} \\
\Phi_{3,1} & \Phi_{3,2} & \lambda_{3} \Phi_{3,1} & \lambda_{3} \Phi_{3,2} & \lambda_{3}^{2} \Phi_{3,2} \\
\Phi_{4,1} & \Phi_{4,2} & \lambda_{4} \Phi_{4,1} & \lambda_{4} \Phi_{4,2} & \lambda_{4}^{2} \Phi_{4,2}
\end{array} \mid .
\end{aligned}
$$

Note that the above determinant representation is given by solving following algebraic equations,

$$
T_{2}=\left.T_{2}\left(\lambda ; \lambda_{1}, \lambda_{2}, \lambda_{3}, \lambda_{4}\right)\right|_{\lambda=\lambda_{j}}\left(\begin{array}{c}
\Phi_{j, 1} \\
\Phi_{j, 2}
\end{array}\right)=0,
$$

where $j=1,2,3,4$. Furthermore the new solution after the two-fold Darboux transformation of Kundu-NLS equations will be

$$
Q^{|2|}=Q+\frac{2 i}{\sqrt{\alpha} e^{i \theta}}\left(t_{1}^{[2]}\right)_{12},
$$

and $\left(t_{1}^{[2]}\right)_{12}=-\left(t_{1}^{[2]}\right)_{21}^{*}$ must hold for $t_{1}^{[2]}$. Then we start with one of the above as a seeding solution and use it with Darboux transformations to obtain more complicated ones.

\section{Rouge Waves of Kundu-NLS Equation}

It is well known that solitons and positon solutions have been generated through Darboux transformation by assuming constant trivial solutions, while the plane-wave solution results in the hierarchy of solutions related to modulation instability. Rational solutions have never been constructed in this way. In order to get rouge waves, we must obtain Akhmediev breathers or Ma solitons. Now, we can take a periodic seed solutions as $Q=c e^{i \rho}$, $\rho=a x+b t, a, b, c$ are arbitrary real constants. Then we can choose $\theta=x$. Substitute the periodic seed solutions into equation.(1.1), we can obtain a constraint relationship, $b+a^{2}-2 \alpha c^{2}+1+2 a=0$. Define

$$
K:=\sqrt{-1-4 \alpha-2 a-4 \lambda^{2}-4 \lambda a-a^{2}-4 \alpha c^{2}},
$$

then the following wave function $\phi_{1}=\left(\begin{array}{l}\phi_{1,1} \\ \phi_{1,2}\end{array}\right), \phi_{2}=\left(\begin{array}{l}\phi_{2,1} \\ \phi_{2,2}\end{array}\right)$ is obtained in the form

$$
\begin{gathered}
\Phi_{1,1}=k_{1} e^{\frac{1}{2} i(\rho+x)-K\left(\frac{1}{2} x+\left(\lambda-\frac{\alpha}{2}-\frac{1}{2}\right) t+\Psi(\varepsilon)\right)}, \\
\Phi_{1,2}=\frac{(i+2 i \lambda+a i-K) k_{1}}{2 \sqrt{\alpha} c} e^{-\frac{1}{2} i(\rho+x)-K\left(\frac{1}{2} x+\left(\lambda-\frac{\alpha}{2}-\frac{1}{2}\right) t+\Psi(\varepsilon)\right)}, \\
\Phi_{2,1}=k_{1} e^{\frac{1}{2} i(\rho+x)+K\left(\frac{1}{2} x+\left(\lambda-\frac{\alpha}{2}-\frac{1}{2}\right) t+\Psi(\varepsilon)\right)}, \\
\Phi_{2,2}=\frac{(i+2 i \lambda+a i+K) k_{1}}{2 \sqrt{\alpha} c} e^{-\frac{1}{2} i(\rho+x)+K\left(\frac{1}{2} x+\left(\lambda-\frac{\alpha}{2}-\frac{1}{2}\right) t+\Psi(\varepsilon)\right)}
\end{gathered}
$$

Here, $\Psi(\varepsilon)=\sum_{k=1}^{n} w_{k-1} \varepsilon^{\frac{2 k-1}{2}}, n$ denotes the number of the steps of the multi-fold Darboux transformation, $w_{k-1} \in C$ are some free parameters and $\varepsilon$ is a infinitesimal parameter. We would like to construct more complicated wave functions to derive more meaningful solutions in the following part, so we mix these four series of wave functions together to derive new functions $\Phi_{1,1}$ and $\Phi_{1,2}$ as follows,

$$
\begin{aligned}
& \Phi_{1,1}:=\Phi_{1,1}(\lambda)-\Phi_{1,2}\left(\lambda^{*}\right)^{*}+\Phi_{2,1}(\lambda)-\Phi_{2,2}\left(\lambda^{*}\right)^{*}, \\
& \Phi_{1,2}:=\Phi_{1,1}\left(\lambda^{*}\right)^{*}+\Phi_{1,2}(\lambda)+\Phi_{2,1}\left(\lambda^{*}\right)^{*}+\Phi_{2,2}(\lambda) .
\end{aligned}
$$

It can be proved that $\Phi_{1,1}$ and $\Phi_{1,2}$ are also the solution of Lax equations with spectral parameter $\lambda$. Using these two wave functions $\Phi_{1,1}$ and $\Phi_{1,2}$, the onefold Darboux transformation will lead to the construction of breather solutions. Next, substituting $\lambda=\xi_{1}+\eta_{1} i$ into $K$, and letting $K=0$, thus we can obtain $\xi_{1}=-(a+1) / 2$, $\eta_{1}=\sqrt{\alpha} c$. If we do the Taylor expansion to breather solutions of the Kundu-NLS equation around $\eta_{1}=\sqrt{\alpha} c$, 
$\xi_{1}=-(a+1) / 2$, the first order rogue wave solutions of the Kundu-NLS equation will be obtained,

$$
\begin{aligned}
Q_{1-r w}= & c e^{i \rho}\left[-16 \alpha c^{2}\left(a^{2}+2 a+\alpha c^{2}+1\right) t^{2}+16(i+x+\right. \\
& \left.a x) \alpha c^{2} t+3-4 \alpha c^{2} x^{2}\right] /\left[16 \alpha c^{2}\left(a^{2}+2 a+\alpha c^{2}+1\right) t^{2}\right. \\
& \left.-16 \alpha c^{2} x(a+1) t+4 \alpha c^{2} x^{2}+1\right] .
\end{aligned}
$$

When we take $a=-1.25, \alpha=1, c=0.75$, the graph for this first order rogue wave solutions of the Kundu-NLS equation and corresponding density graph are shown in Figure 1.

However, it is highly non-trivial to construct higher rogue waves from the higher order breathers because of the multi-degeneration of the eigenvalues. Similar to the case of the NLS equation, the determinant representation of the Darboux transformation provides a useful tool to calculate this tedious expansion. Value $\lambda=-(a+1) / 2$ $+i \sqrt{\alpha} c$ is a zero point of the eigenfunction $\Phi_{1,1}, \Phi_{1,2}$, and all $\lambda_{j}=\lambda+\varepsilon$ with $\varepsilon$ denoting a small parameter when we consider the degeneration of the eigenfunction. Therefore, let the first and second rows in $Q^{[2]}$ expand into the second power to $\varepsilon$ and the third and forth rows expand into the third power to $\varepsilon$. By the two-fold Darboux transformation, we can obtain the second order rogue wave solutions of the Kundu-NLS equation. Here, we can only draw the graph the second order rogue wave solutions of the Kundu-NLS equation in Figure 2. Similarly, we can obtain the third order rogue wave solutions of the Kundu-NLS equation.Choose $a=-1.25, \quad c=0.4$, $\alpha=1$, the graph of the third order rogue wave solutions of Kundu-NLS equation is drawn in Figure 3.

Until now, from the first order rogue wave to the third order rogue wave, basic modes have been given when $w_{k-1}=0(k \geq 1)$. But $w_{k-1}$ has different values in different $\Phi_{1,1}$ and $\Phi_{1,2}$. Next we discuss the impact about $w_{k-1}$ to the higher order rogue waves. To the second order rogue wave solutions, $\Psi(\varepsilon)$ have two free para- meters for the Kundu-NLS equation. We choose $w_{0}=0$, $w_{1}=200$, the second order rogue wave is well separated into three single rogue waves. These single rogue waves exhibit a triangular shape. The graph is shown in Figure 4. Similar behavior is obtained using the third order rogue wave solution, $\Psi(\varepsilon)$ have three free parameters. Chooing $a=-1.25, c=0.43, \alpha=1, \quad w_{0}=0, w_{1}=10, w_{2}=0$, the third order rogue wave is well separated into six single rogue waves, which perform in a triangular shape. Then we choose $a=-1, c=0.5, \alpha=1, w_{0}=0, w_{1}=0$, $w_{2}=1000$, the third order rogue wave is composed of six single rogue waves, which form a pentagon with one peak in the center and the rest are located on the vertices of the pentagon. Figure 5 and Figure 6 illustrate the corresponding combination of different types of the third order rogue wave.

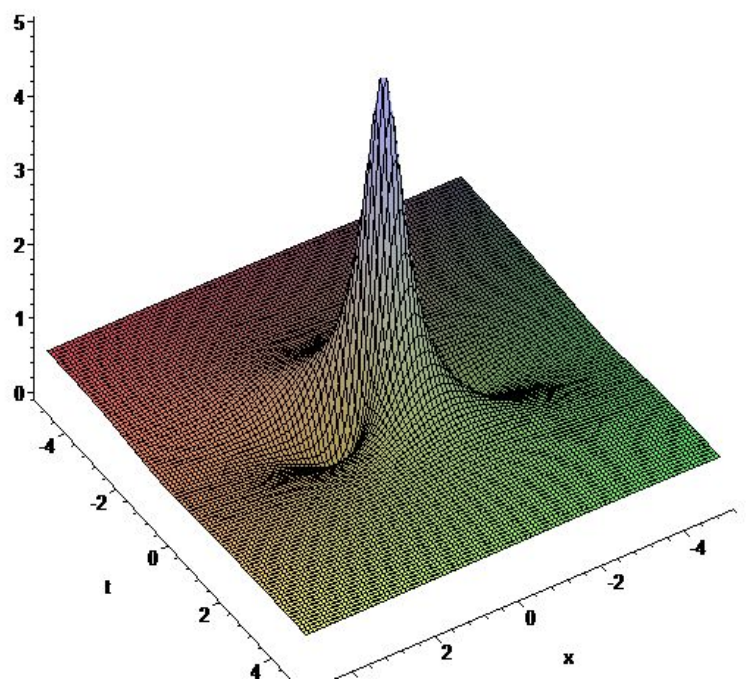

Figure 1. The first order rogue wave solution $Q$.

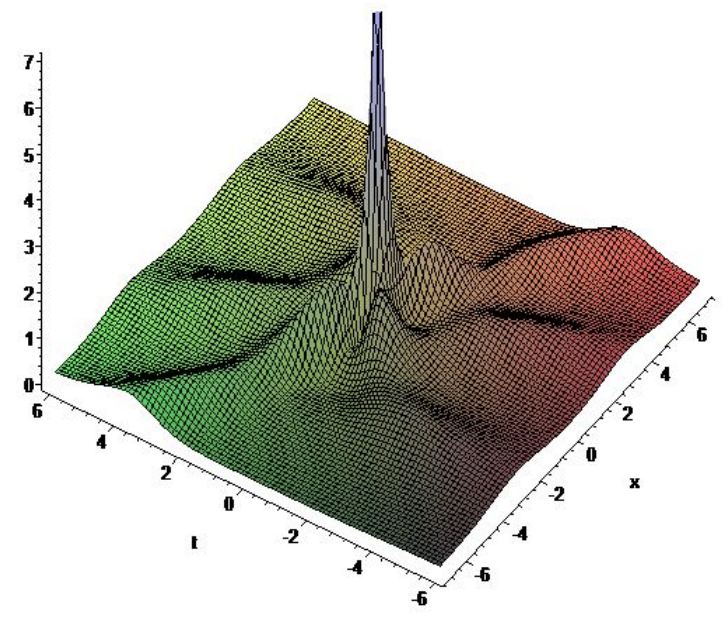

Figure 2. The second order rogue wave solution $Q$ when $a=$ $-1.25, \alpha=1, c=0.43$.

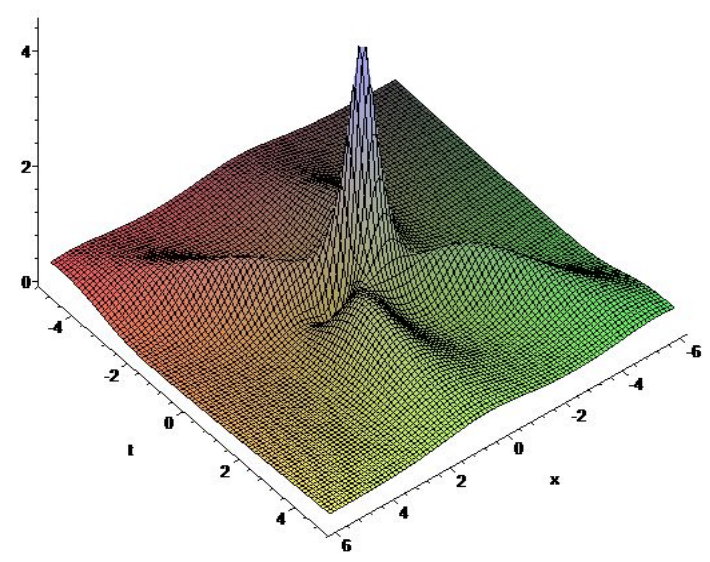

Figure 3. The third order rogue wave solutions $Q$ when $a=$ $-1.25, c=0.4, \alpha=1$, when $a=-1.25, \alpha=1, c=0.75$. 


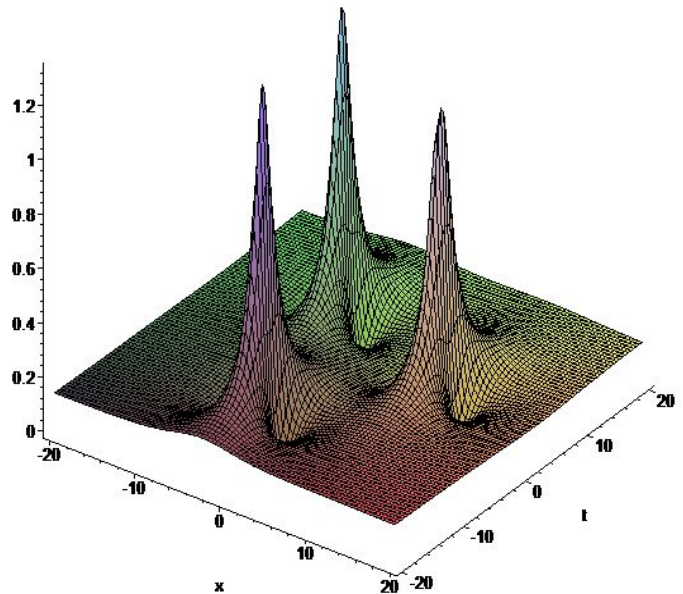

Figure 4. The second rogue wave solution $Q$ when $a=-1, c$ $=0.375, \alpha=1, w_{0}=0, w_{1}=200$.

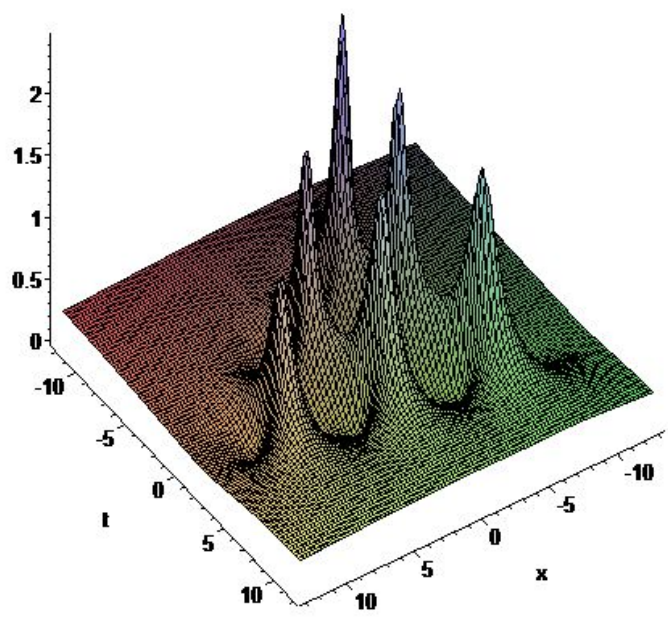

Figure 5. The third order rogue wave solutions $Q$ when $a=$ $-1.25, c=0.43, \alpha=1, w_{0}=0, w_{1}=10, w_{2}=0$.

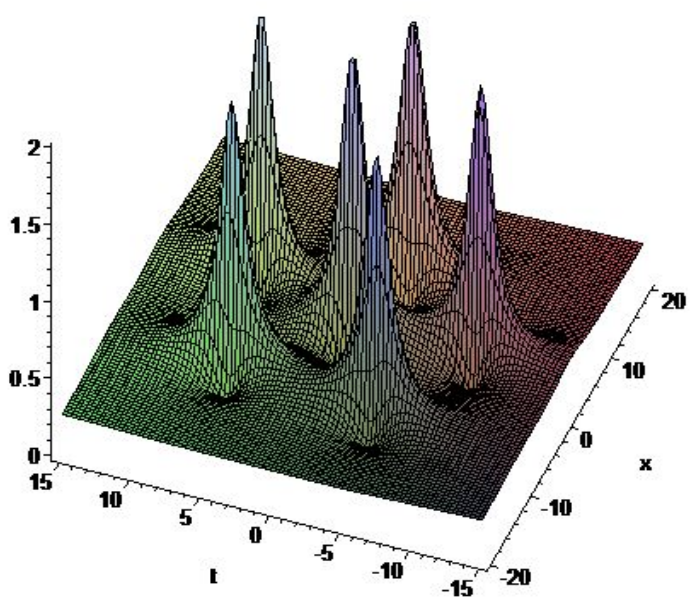

Figure 6. The third rogue wave solution $Q$ when $a=-1, c=$ $0.5, \alpha=1, w_{0}=0, w_{1}=0, w_{2}=1000$.
Viewing in Figure 4, Figure 5 and Figure 6, we can say that some free parameters in $\Psi(\varepsilon)$ play a role in eigenfunctions for the Kundu-NLS equation. Adjusting the value of $w_{1}$, the higher order rogue waves can be separated into some single rogue waves, and these firstorder rogue waves exhibit a triangular shape. What is more, letting $w_{1}$ value be bigger, the triangular shape will be separated more obviously. Particularly, there exists other shapes of the third order rogue waves, but they must satisfy $w_{0}=0, w_{1}=0$, meanwhile a pentagon can be obtained by adjusting the value of $w_{2}$.

\section{Summary}

In this paper, based on the Darboux transformation of the Kundu-NLS equation, the rogue wave solutions of the Kundu-NLS equation are constructed explicitly from periodic seed solutions. Some free parameters in eigenfunctions can adjust shapes of the higher order rogue waves such that it can be separated into some single rogue waves. These may have very important meaning in physics and it deserves further studying.

\section{Acknowledgements}

This work is supported by the NSF of China under Grant No.10971109 and No.11271210 and K.C.Wong Magna Fund in Ningbo University. Jingsong $\mathrm{He}$ is also supported by Natural Science Foundation of Ningbo under Grant No.2011A610179.

\section{REFERENCES}

[1] V. B. Matveev and M. A. Salle, "Darboux Transformations and Solitons," Springer, Berlin, 1991, pp. 113-115. doi: 10.1007/978-3-662-00922-2

[2] J. S. He, L. Zhang, Y. Cheng and Y. S. Li, "Determinant Representation of Darboux Transformation for the AKNS System," Science in China Series A: Mathematics, Vol. 49, No. 12, 2006, pp. 1867-1878. doi: $10.1007 / \mathrm{s} 11425-006-2025-1$

[3] T. Waseda, "Rogue Waves in the Ocean," Eos, Transactions American Geophysical Union, Vol. 91, No. 11, 2010, p. 104. doi: 10.1029/2010EO110007

[4] D. R. Solli, C. Ropers, P. Koonath and B. Jalali, "Optical Rogue Waves," Nature, Vol. 450, 2007, pp. 1054-1057. doi: 10.1038 /nature06402

[5] A. Chabchoub, N. P. Hoffmann and N. Akhmediev, "Rogue Wave Observation in a Water Wave Tank," Physical Review Letters, Vol. 106, No. 20, 2011, pp. 204-502. doi: 10.1103/PhysRevLett.106.204502

[6] Y. V. Bludov, V. V. Konotop and N. "Akhmediev, "Matter Rogue Waves," Physical Review A, Vol. 80, No. 3, 2009, p. 033610. doi: 10.1103/PhysRevA.80.033610

[7] L. Wen, L. Li, Z. D. Li, S. W. Song, X. F. Zhang and W. M. Liu, "Matter Rogue Wave in Bose-Einstein Conden- 
sates with Attractive Atomic Interaction," The European Physical Journal D, Vol. 64, No. 2-3, 2011, pp. 473-478. doi: 10.1140/epjd/e2011-20485-4

[8] M. S. Ruderman, "Freak Waves in Laboratory and Space Plasmas," The European Physical Journal, Vol. 185, No. 1,2010, pp. 57-66. doi: 10.1140/epjst/e2010-01238-7
[9] Z. Yan, "Financial Rogue Waves," Communications in Theoretical Physics, Vol. 54, No. 4, 2010, pp. 947-949. doi: $10.1088 / 0253-6102 / 54 / 5 / 31$

[10] J. S. He, H. R. Zhang, L. H. Wang, K. Porsezian and A. S. Fokas. "A Generating Mechanism for Higher Order Rogue Waves,". 